\title{
Dual inhibition of EGFR and VEGFR pathways in combination with irradiation: antitumour supra-additive effects on human head and neck cancer xenografts
}

\author{
A Bozec', P Formento', S Lassalle ${ }^{2}$, C Lippens', P Hofman ${ }^{2}$ and G Milano ${ }^{*, 1}$ \\ 'Oncopharmacology Unit, Centre Antoine-Lacassagne, Nice, France; ${ }^{2}$ Department of Pathology, University Hospital, Nice, France
}

The aim of this study was to investigate the effects of combining antiangiogenic treatment, epidermal growth factor receptor (EGFR) targeting and irradiation (RT). We evaluated AZD2 I7I, a highly potent, orally active, vascular endothelial growth factor (VEGF) signalling inhibitor, gefitinib, an EGFR tyrosine kinase inhibitor and RT. The antitumour efficacy of these treatments, administered alone and in combination for 2 weeks, was assessed in a VEGF-secreting human head and neck tumour cell line, CAL33 that highly expresses EGFR, established as xenografts $\left(250 \mathrm{~mm}^{3}\right)$ in nude mice. The median time to reach a tumour volume of $1000 \mathrm{~mm}^{3}$ was significantly increased for AZD2 17I or gefitinib alone compared with the control. Greater inhibition of tumour growth was seen with the combination of AZD2I7I + gefitinib compared with either drug alone, and the triple combination compared with either AZD2I7I + gefitinib or RT alone. The intensity of endothelial cell staining was slightly reduced by each agent given alone, and markedly diminished by the double or triple combination. The triple combination almost completely abolished cell proliferation. The marked RT-induced enhancement in the DNA-repair enzyme ERCCI expression was totally abolished by the triple combination. This observation could help to explain the supra-additive antitumour effect produced by this combination and could provide a basis for future innovative clinical trials.

British Journal of Cancer (2007) 97, 65-72. doi:10.1038/sj.bjc.660379I www.bjcancer.com

Published online 26 June 2007

(c) 2007 Cancer Research UK

Keywords: AZD217I; gefitinib; radiotherapy; head and neck cancer; antiangiogenic; tyrosine kinase inhibitor

Agents that target epidermal growth factor receptor (EGFR) potentially exert antitumour effects by inhibiting tumour cell proliferation and survival, as well as reducing the secretion of proangiogenic growth factors such as vascular endothelial growth factor (VEGF) and fibroblast growth factor that stimulate tumour neoangiogenesis (Perrotte et al, 1999; Woodburn, 1999; Ciardiello et al, 2001). It has been reported that EGFR targeting in tumours may also modulate the migration and formation of tube-like structures of vascular endothelial cells (Hirata et al, 2002). More recently, we have shown the presence of a gefitinib-sensitive functional EGFR pathway in an immortalised microvascular endothelial cell line of human origin (Bozec et al, 2005). Thus, in addition to direct effects on tumour cells, EGFRtargeting drugs may also impart an indirect antitumour effect through antiangiogenic activity. An optimal antiangiogenic strategy may, therefore, be to combine an EGFR signalling inhibitor with an agent that selectively targets VEGF-dependent signalling.

\footnotetext{
*Correspondence: Dr G Milano, Oncopharmacology Unit, Centre Antoine-Lacassagne, 33 Avenue de Valombrose, 06189 Nice Cedex 2, France; E-mail: gerard.milano@nice.fnclcc.fr

Revised 16 April 2007; accepted 19 April 2007; published online 26 June 2007
}

The novel indole-ether quinazoline, AZD2171, is a highly potent $\left(\mathrm{IC}_{50}<1 \mathrm{nM}\right)$ ATP-competitive inhibitor of recombinant VEGF receptor-2 (VEGFR-2) tyrosine kinase in vitro (Wedge et al, 2005). AZD2171 also shows potent activity $v s$ VEGFR-1 $\left(\mathrm{IC}_{50}=5 \mathrm{nM}\right)$ and VEGFR-3 ( $\left.\mathrm{IC}_{50} \leqslant 3 \mathrm{nM}\right)$. In human umbilical vein endothelial cells, AZD2171 inhibited VEGF-stimulated proliferation and VEGFR-2 phosphorylation with $\mathrm{IC}_{50}$ values of 0.4 and $0.5 \mathrm{nM}$, respectively. In a fibroblast/endothelial cell coculture model of vessel sprouting, AZD2171 reduced vessel area, length and branching at subnanomolar concentrations. The growth of established human tumour xenografts in athymic mice was dose-dependently inhibited by chronic administration of AZD2171. Combining AZD2171 and gefitinib could potentially provide inhibitory effects on both endothelial and tumour cells. Recent preclinical studies suggest that radiotherapy (RT) in combination with antiangiogenic/vasculature-targeting agents may enhance the therapeutic ratio of ionising radiation alone (Wachsberger et al, 2005; Cao et al, 2006; Williams et al, 2007). Thus, the combination of AZD2171 and gefitinib with RT could also be interesting to investigate.

Tumour vasculature is a key target in the treatment of solid tumours, particularly in head and neck cancer (Le and Giaccia, 2003). In the present study, the antitumour activity of AZD2171, in combination with gefitinib and RT on a number of cellular and molecular markers in human head and neck tumour xenografts (CAL33), was investigated. 


\section{MATERIALS AND METHODS}

\section{Chemicals}

AZD2171 and gefitinib were kindly provided by AstraZeneca, Macclesfield, Great Britain. Working solutions were prepared as follows: AZD2171 $\left(375 \mathrm{mgl}^{-1}\right)$ and gefitinib $\left(7.5 \mathrm{gl}^{-1}\right)$ were suspended in $0.9 \% \mathrm{NaCl}, 0.01 \%$ Tween 80 . For both drugs, the concentrations were adjusted so as to include the daily dose in $0.2 \mathrm{ml}$ of drug suspension. Dulbecco's modified Eagle's medium (DMEM), penicillin, streptomycin and glutamine were purchased from Whittaker (Verviers, Belgium). Foetal bovine serum (FBS) was obtained from Dutscher (Brumath, France).

\section{Cell lines}

CAL33, a cell line of human head and neck origin, was obtained from our institution (Centre Antoine-Lacassagne, Nice, France). This cell line exhibits high EGFR levels $\left(33794 \pm 624 \mathrm{fmol} \mathrm{mg}^{-1}\right.$ protein high-affinity sites determined by ligand binding assay; Dassonville et al, 1993) and produces VEGF (C Onesto, CNRSUMR6543, personal communication).

The cell line was maintained as monolayer culture in DMEM supplemented with $10 \% \mathrm{FBS} \mathrm{v} / \mathrm{v}, 2 \mathrm{~mm}$ glutamic acid, $50000 \mathrm{Ul}^{-1}$ penicillin and $80 \mu \mathrm{m}$ streptomycin in a humidified incubator (Sanyo, Osaka, Japan) at $37^{\circ} \mathrm{C}$ in an atmosphere containing $8 \%$ $\mathrm{CO}_{2}$. Batches of $15 \times 10^{6}$ cells were frozen in FBS supplemented with $5 \%$ dimethyl sulphoxide $(\mathrm{v} / \mathrm{v})$ in advance for injection into mice. Shortly before injection, cells were thawed and suspended in Ringer lactate.

\section{Mice}

Animal experiments were performed in accordance with the regulations of our institution's ethics commission and with the United Kingdom Co-ordinating Committee on Cancer Research guidelines (Workman et al, 1998). Six-week-old male NMRI nude mice were purchased from Janvier laboratories (Le Genet sur Isle, France) and received s.c. inoculation in the right flank of $2 \times 10^{6}$ cells suspended in $100 \mu$ l of Ringer lactate $(n=10$ per treatment condition). There were five animals per cage with food and water ad libitum; following tumour cells injection, animal weight and tumour growth were monitored once a week. Animals were killed at the end of experiment by cervical disruption; as no signs of suffering appeared during the experiment (submitted attitude, weight loss, prostration, vocalisation), no animal has to be killed before the end of experiment.

\section{Treatment}

Mice bearing well-established CAL33 tumours (mean tumour volume/treatment group $\sim 250 \mathrm{~mm}^{3}$ ) were treated each week with vehicle alone (controls), AZD2171 $\left(2.5 \mathrm{mg} \mathrm{kg}^{-1}\right.$ every day $0.2 \mathrm{ml}$ p.o.), gefitinib ( $50 \mathrm{mg} \mathrm{kg}^{-1} \mathrm{day}^{-1}, 5$ days per week, $0.2 \mathrm{ml}$ p.o.) and RT (6 Gy day ${ }^{-1}, 3$ days per week, $2 \mathrm{~h}$ after drugs administration) for 2 weeks. When coadministered, AZD2171 and gefitinib were given simultaneously. The dose of gefitinib and AZD2171 were chosen according to preliminary experiments so that each drug given alone exerts only partial effects on tumour growth.

Radiotherapy was performed with $\gamma$-rays on tumour only, using a ${ }^{60} \mathrm{Co}$ unit, the animals being maintained and biologically isolated from ambient air during RT with a straitjacket made of Saran wrap film.

The effect of AZD2171 and gefitinib alone or in combination with RT on tumour growth was evaluated. The effects of the treatments were calculated, as described previously (Prewett et al, 2002). Evaluation of the effects on tumour growth consisted in measuring, for all groups, the mean tumour volume at the end of the observation period for the controls (day 30), when tumours in this group reached the average volume of $2500 \mathrm{~mm}^{3}$ (maximal ethically acceptable volume). Fractional tumour volume (FTV) for each treatment group was calculated as the ratio between the mean tumour volumes of treated and untreated animals. This was performed for treatment a (FTVa), for treatment $b$ (FTVb) and for treatment $a+b($ FTV $a+b)$. The expected FTV for the $a+b$ combination was defined as FTVa observed $\times$ FTVb observed. The ratio FTVa $+\mathrm{b}$ expected/FTVa $+\mathrm{b}$ observed was the combination ratio (CR). If $C R>1$, there are supra-additive effects and if $C R<1$ infra-additive ones. Strictly additive effects were observed if $\mathrm{CR}=1$. Another way to compare treatment effects was to calculate the time necessary for the tumours to reach a volume of $1000 \mathrm{~mm}^{3}$ (log-rank test).

\section{Tumour analysis}

Tumour length and width was measured weekly using a calliper rule. Tumour volume was calculated as $\pi / 6 \times$ length $\times$ width $^{2}$ until animal killing. Animals were killed by spinal cord dislocation and tumours were subsequently removed surgically. Half of the tumour was directly frozen in liquid nitrogen for protein analysis and the other half fixed in paraformaldehyde overnight for analysis of the microvessel marker, von Willebrand factor (vWF; Dako, polyclonal antibody ref.: A0082), and the proliferation marker, Ki67 (Dako, Trappes, France, monoclonal antibody ref.: M7240, MIB-1), using immunohistochemistry after tumour tissue from the experimental groups was assembled into microtissue arrays (Simon et al, 2004). The analysis of vWF concerned both vessel loss and reduction in vessel area. The analysis of Ki67 took into account both the intensity of labelling and proportion of labelled cells.

The final score was the result of the examination of three fields per tumour, and between four and five tumours were investigated for each treatment group. One hundred $\times 10$ magnification images of regions of high vascular density within the tumour were analysed in order to quantify tumour angiogenesis (vWF). Staining intensities were scored as: $1=$ slight; $2=$ medium; and $3=$ strong. A subgroup $(n=5)$ was killed on day 23 (the last day of treatment) and tumours collected, while the remaining animals $(n=5)$ were killed in each group, once animals in the group reached a tumour volume of $2500 \mathrm{~mm}^{3}$. Approximately, these times were reached on day 30 for control and gefitinib, on day 37 for AZD2171 and AZD2171 + gefitinib, on day 50 for RT, and on day 60 for AZD2171 + gefitinib + RT treatment groups.

Frozen tumours were pulverised in a liquid nitrogen-cooled Thermovac pulveriser. The resulting powders were homogenised in 10 volumes of a $10 \mathrm{~mm}$ Tris- $\mathrm{HCl}$ buffer $\mathrm{pH} 7.4$, containing $1 \mathrm{~mm}$ EDTA, $0.5 \mathrm{~mm}$ dithiothreitol, $10 \mathrm{~mm}$ sodium molybdate, phosphatase inhibitor cocktail 2 with a dilution $1 / 100$ and protease inhibition cocktail 2 with a dilution 1/100, both from Sigma (Saint Quentin Fallavier, France). The homogenates were centrifuged for $1 \mathrm{~h}$ at $105000 \mathrm{~g}\left(+4^{\circ} \mathrm{C}\right)$ and the supernatants (cytosols) were used for protein determination by immunoblotting. Total protein content was measured using the bicinchoninic acid assay.

Human VEGF secreted by CAL33 xenografted tumours was determined in tumour cytosol by ELISA using DVE00 (Quantikine, R\&D systems, Lille, France). Epidermal growth factor receptor had been previously determined by the $\mathrm{I}^{125}$-EGF-binding method followed by Scatchard-plot analysis (Dassonville et al, 1993).

The EGFR and VEGFR signalling pathway markers phosphoERK1/2, PTEN and phospho-AKT, the apoptosis-related markers $\mathrm{Bax} / \mathrm{Bcl} 2$ ratio, the proliferation-related marker $\mathrm{p} 27$ and the DNA repair-related marker ERCC1 were determined by Western blot (detailed technical conditions are summarised in Table 1).

Two sets of tumours have been analysed with different purposes. One set (five animals per treatment group) was collected at the end of treatment period (day 23) in order to compare the effects of the different treatments on a panel of molecular markers. The other set 
Table I Technical conditions of Western blot analyses

\begin{tabular}{lclll}
\hline Antibody & \% Acrylamide & Dilution & Source & Supplier \\
\hline PTEN & 12 & I//000 TBS 5\% milk & Mouse & Pharmingen \\
ERCCI & 12 & I/500 TBS 5\% milk & Mouse & Neomarkers \\
Phospho-AKT & 12 & I/I000 TBS 5\% BSA & Rabbit & Ozyme \\
P27 & 12 & I//000 TBS 5\% milk & Rabbit & Pharmingen \\
Bax & 12 & I//000 TBS 5\% milk & Rabbit & Upstate \\
Bcl2 & 12 & I//000 TBS 5\% milk & Rabbit & Pharmingen \\
Phospho-ERKI/2 & 12 & I/5000 TBS 5\% milk & Mouse & Sigma \\
\hline
\end{tabular}

$\mathrm{BSA}=$ bovine serum albumin; TBS = tris-buffered saline.

of tumour-bearing animals was maintained to assess tumour regrowth after the end of treatment period. Animals were killed when mean tumour volume of each treatment group reached $2500 \mathrm{~mm}^{3}$. These tumours were used to detect what molecular parameters could be modified in these tumours escaping to treatments. The first set of tumours was analysed for vWF (endothelial cell-specific marker) and Ki67 (proliferative capacity of tumour cells) by immunohistochemistry, for the EGFR and VEGFR signalling pathway markers such as phospho-ERK1/2 and phospho-AKT, for the apoptosis-related markers $\mathrm{Bax} / \mathrm{Bcl} 2$ ratio, the proliferation-related markers p21 and p27 and the DNA repairrelated marker ERCC1 (essentially on tumour cells as they largely outnumber endothelial cells in tumours) by Western blot normalised by Raf as a loading control, and VEGF (human, secreted by tumour cells only) by ELISA.

The second set of tumours was analysed for phospho-ERK1/2, phospho-AKT and PTEN expression by Western blot also normalised by Raf as a loading control.

The bands in the Western blots were quantified using the Chemi Doc imager from Bio-Rad, Marnes-La-Coquette, France.

\section{Statistical analyses}

Comparison of tumour growth between different treatment groups was performed by Kaplan-Meier-type analysis with the log-rank statistical test. The effects of treatment on vWF and Ki67 were evaluated using the non-parametric ANOVA (Kruskal-Wallis test). The differences between treatment groups for molecular factors (VEGF, phospho-ERK1/2, PTEN, phospho-AKT, Bax/Bcl2, ERCC1) were examined using the Mann-Whitney test.

\section{RESULTS}

\section{Effects of AZD2171 in combination with gefitinib and radiation on tumour growth}

The effect of the different treatments on tumour growth is shown in Figure 1. Tumour growth was inhibited by AZD2171 or gefitinib given alone. These antitumour effects were rapidly established but disappeared after treatment cessation. In contrast, the antitumour effects of RT were only seen after 1 week of treatment and persisted over a longer period. Combination treatment with AZD2171+ gefitinib produced a greater inhibition of tumour growth than either treatment alone and led to a growth arrest. Tumour regrowth occurred after the end of treatment in all groups with either drugs alone or when combining AZD2171 with gefitinib.

We then considered the AZD2171-gefitinib combination as a single-drug treatment and examined the effects of its combination associated with RT. The triple combination (AZD2171+ gefitinib + RT) produced a greater antitumour effect than either RT or the AZD2171 - gefitinib combination. In comparison to RT or to the AZD2171 - gefitinib combination, the triple combination prolonged the antitumour effects after treatment discontinued.

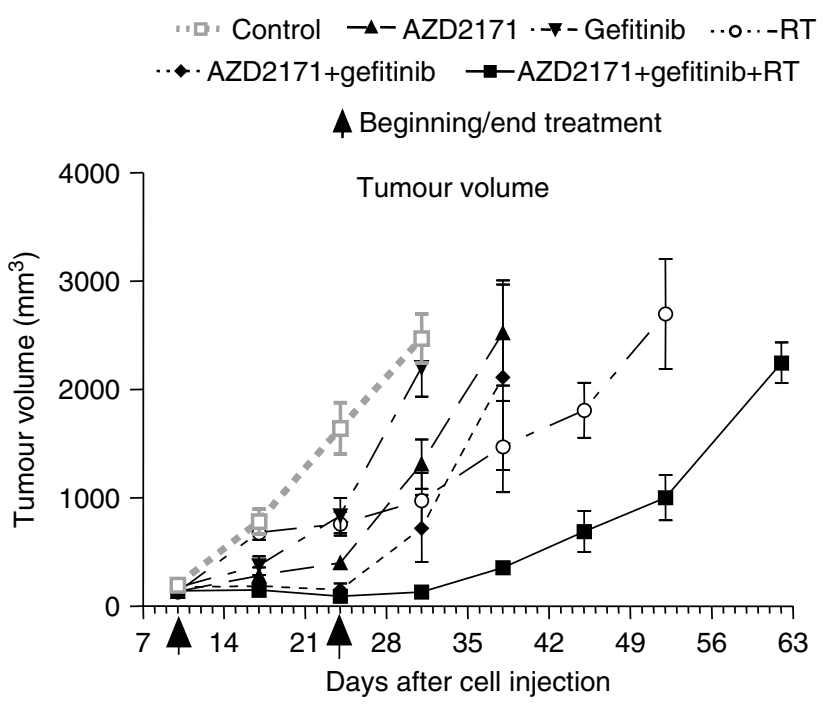

Figure I Effects of AZD2 I7I (2.5 mg kg-1, every day during 2 weeks), gefitinib (50 $\mathrm{mg} \mathrm{kg}^{-1}, 5$ days per week during 2 weeks), RT (6 Gy, three times per week, every 2 days during 2 weeks, $2 \mathrm{~h}$ after drugs) or a combination on growth of CAL33 head and neck tumours (mean tumour volume \pm s.d., $n=10$ per treatment condition until day 24 , then $n=5$ per treatment group except for RT where $n=4$ ).
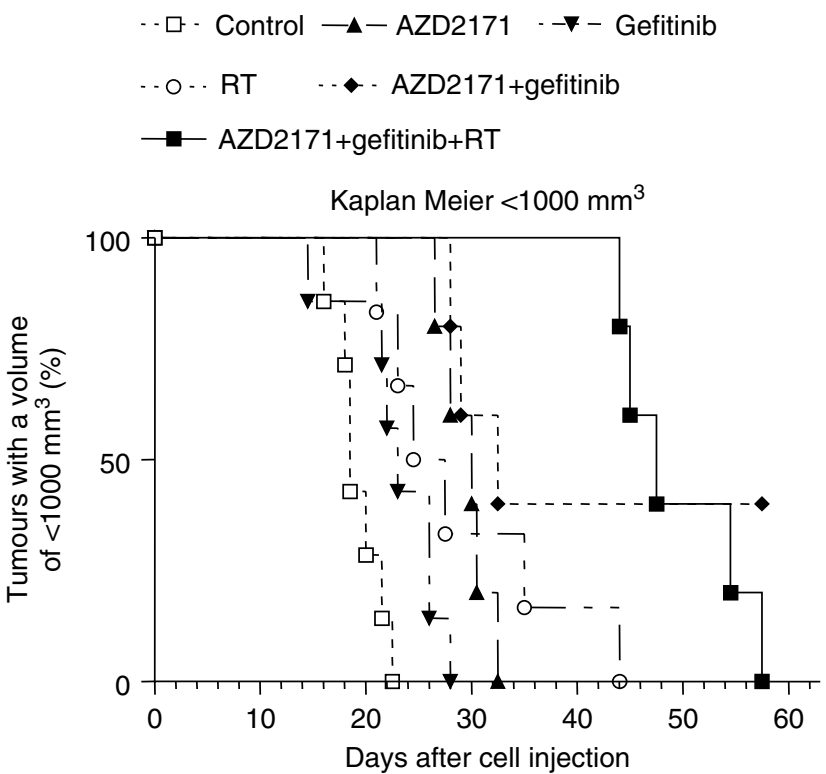

Figure 2 Respective time delay to reach a tumour volume of $1000 \mathrm{~mm}^{3}$ for the different treatment groups (seven mice for control and gefitinib, six mice for RT and five mice for all other treatment groups). Of note, two tumours never reached the $1000 \mathrm{~mm}^{3}$ volume (these tumours were purely necrotic but still present at the end of the experiment).

Following the treatment period with the triple combination, growth arrest lasted for 1 week, followed during 3 weeks by a growth paralleling that of the RT-treated tumours and by a rapid growth during the last 10 days.

Antitumour effects for AZD2171 + gefitinib in combination were supra-additive $(\mathrm{CR}=1.6)$ as were those for the triple combination of both drugs administered with $\mathrm{RT}(\mathrm{CR}=2)$. These $\mathrm{CR}$ values $(>1)$ are consistent with tumour growth inhibition observed with each regimen.

The median time to reach a tumour volume of $1000 \mathrm{~mm}^{3}$ was significantly increased for AZD2171 or gefitinib alone compared 
with the control $(P=0.001)$. There was also a significant difference between the combination of AZD2171 + gefitinib and either drug alone $(P=0.006)$ and between the triple combination and AZD2171 + gefitinib $(P=0.0001)$ or $\quad$ RT alone $\quad(P<0.0001$; Figure 2).

Body weights were measured as surrogate markers of treatment tolerance with a slight body weight loss in all treatment groups compared to the control group, thus suggesting that treatments were well tolerated (Figure 3).

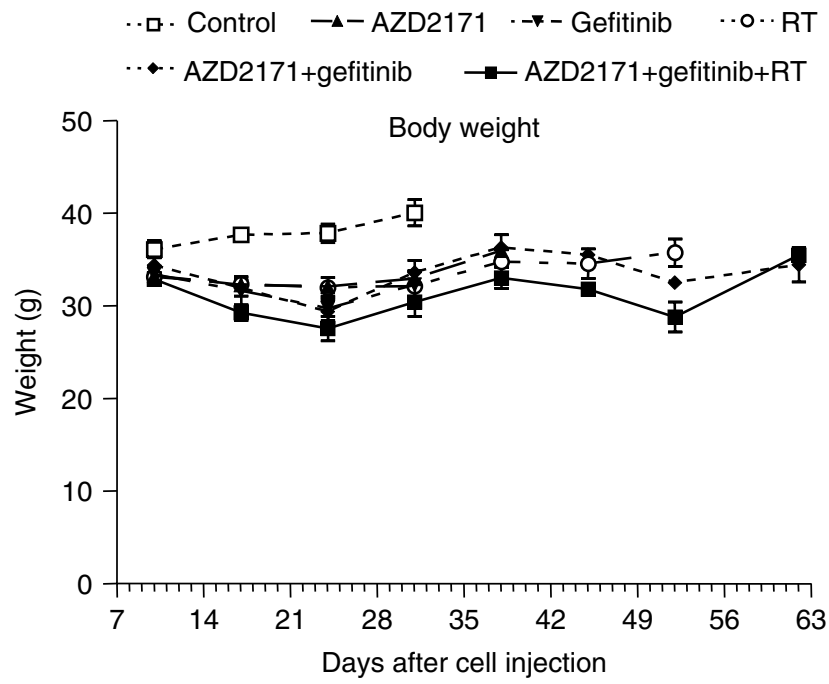

Figure 3 Profile of body weight vs time among the different treatment groups (mean weight \pm s.d., $n=10$ per treatment condition until day 24 , $n=5$ thereafter).

\section{Effects of treatments on molecular parameters}

In the first set of tumours (collected at the end of treatment period), the intensity of vWF staining was slightly reduced by each agent given alone, and markedly diminished by the double or triple combination $(P<0.0001$ for triple association $v s$ control; Figure 4$)$.

The triple combination almost completely abolished proliferation in tumour cells as shown by the decrease in Ki67 labelling intensity $(P<0.001$ for triple association $v s$ control; Figure 5$)$. The effects on apoptosis-related factors $\mathrm{Bax} / \mathrm{Bcl} 2$ ratio were inconsistent (data not shown). No significant effect on phospho-ERK1/2 and on phospho-AKT was observed with any of the treatment groups (data not shown).

The marked RT-induced enhancement in ERCC1 expression was totally abolished by the triple combination $(P=0.04$ for the triple combination $v s$ RT; Figure 6). This result could help to explain the supra-additive antitumour effect produced by this combination.

Compared with VEGF levels in the control tumours, gefitinib alone tended to decrease VEGF concentration $(P=0.157)$, whereas AZD2171 alone enhanced levels of VEGF in the tumour $(P=0.02$ $v s$ control). The combination AZD2171 + gefitinib as well as RT alone had no effect on VEGF levels, whereas the triple combination was associated with an increase in VEGF (Figure 7).

In addition to measurements of tumour factors at the end of treatment, there were molecular investigations performed in the second set of tumours (obtained at the end of follow-up when tumours had regrown). All tumours were collected at a mean volume of $2500 \mathrm{~mm}^{3}$. There was a variable diminution of phosphoERK1/2 expression as compared to the control for all treatment conditions (data not shown). All the treatment groups showed an increase in phospho-AKT expression with the triple association showing the largest increase $(P<0.0001$; Figure 8$)$. All treatment groups showed a marked decrease in PTEN expression, the phosphorylase, which negatively controls the PI3-Akt pathway (reaching statistical significance for RT and gefitinib + AZD2171 combination only, $P=0.04$ and 0.03 , respectively; Figure 9). These
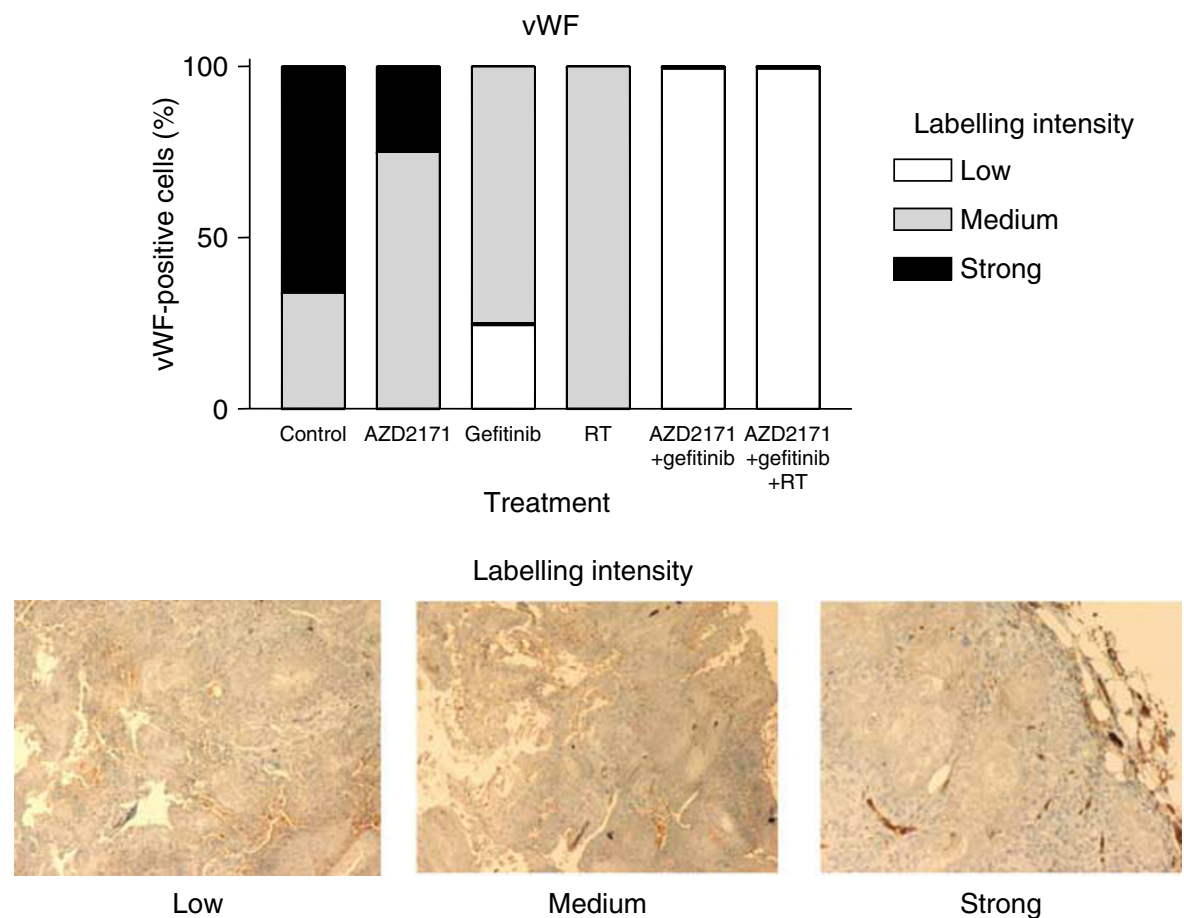

Figure 4 Impact of the different treatments on day 24 at the end of the treatment period for the different treatments on vWF (endothelial cell marker), four microscope fields observed on four tumours (conditions AZD2 I7I, gefitinib, AZD2 I7I-gefitinib), five tumours (conditions RT, AZD2 I7I-gefitinibRT), six tumours (control). Magnification $\times 20$ for labelling intensity. 

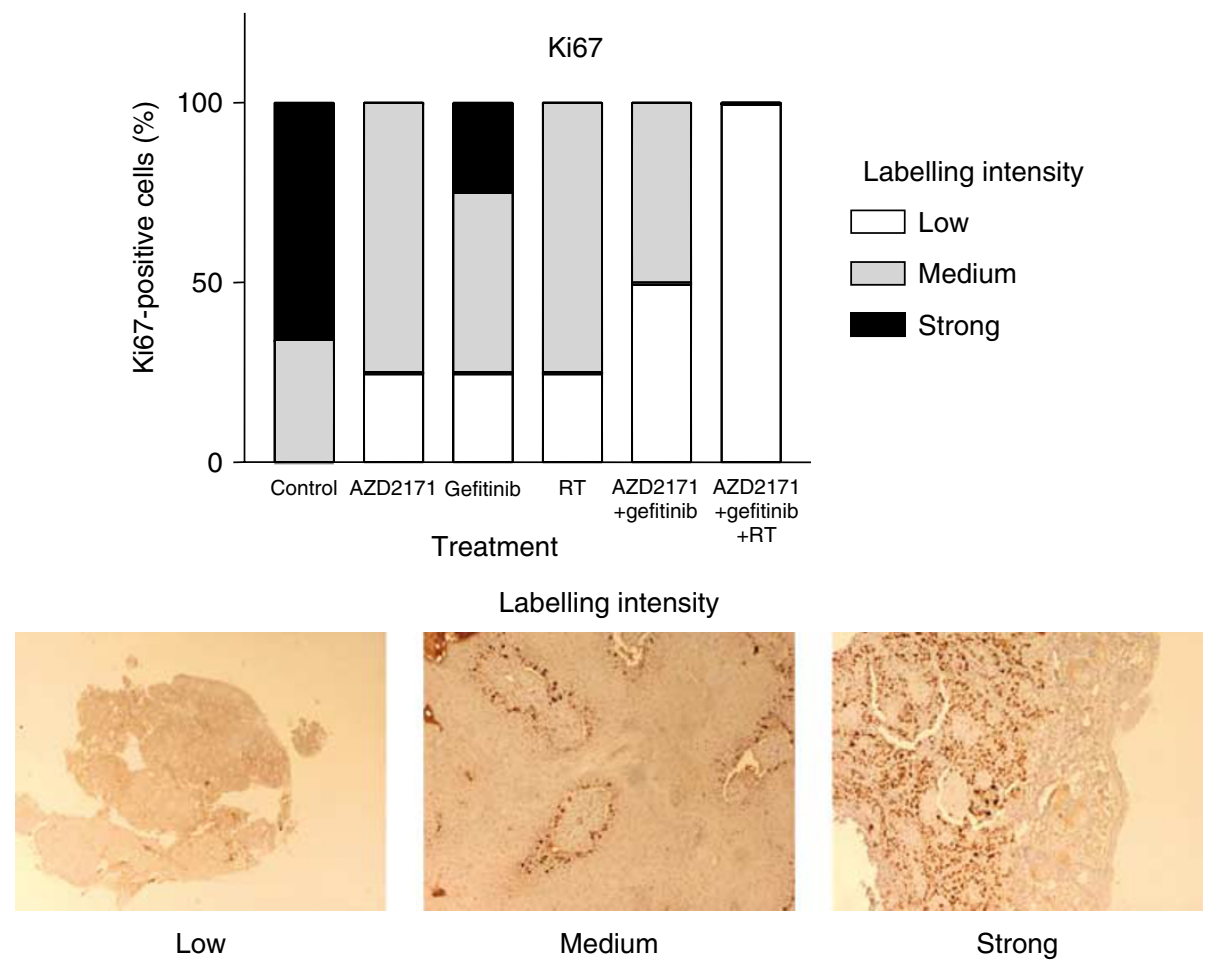

Figure 5 Impact of the different treatments on Ki67 staining (proliferation marker) on day 24 at the end of the treatment period for the different treatments (four microscope fields observed) on four tumours (conditions AZD217I, gefitinib, AZD2I7I-gefitinib), five tumours (conditions RT, AZD2 I I -gefitinib-RT), six tumours (control). Magnification for labelling intensity: $\times 4$ for low and $\times 20$ for medium and strong.
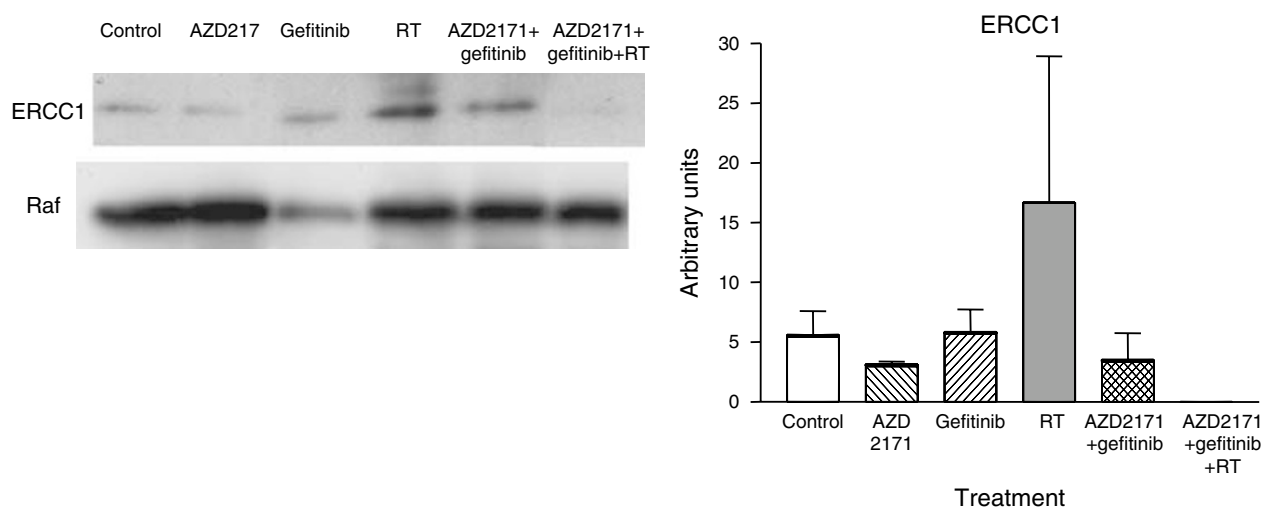

Figure 6 Effect of the different treatments on the expression of ERCCI on day 24 at the end of the treatment period for the different treatments (mean expression \pm s.d.), three tumours for control and AZD2 I7I-gefitinib-RT and four tumours for all other treatment conditions. A typical example of Western blot analysis is given. The results were normalised vs Raf taken as a loading control.

results corroborate the effects observed on phospho-AKT. In addition, all treatments except gefitinib alone showed a marked decrease in the expression of the cell-cycle regulating protein p27 $(P=0.001$; Figure 10$)$. Results with p21 were less consistent (data not shown).

\section{DISCUSSION}

Several preclinical studies have examined the antitumour activity of inhibitors of EGFR and antiangiogenic agents in combination and have demonstrated at least additive, if not synergistic, effects (Ciardiello et al, 2000; Jung et al, 2002; Herbst et al, 2003). These encouraging data have led to the initiation of clinical studies in lung cancer, evaluating the combination of erlotinib, an EGFR tyrosine kinase inhibitor, with bevacizumab, an anti-VEGF antibody (Herbst et al, 2005). Previous experimental studies showed potential beneficial antitumour effects when combining antiangiogenic agents with RT (Wachsberger et al, 2005, Nieder et al, 2006), resulting in at least additive effects on tumour growth delay despite different radiation schedules, drugs and doses and combination regimens. Clinical research in this field is ongoing but additional preclinical studies are needed to further evaluate drug combinations, including the targeting of EGFR and VEGF signalling pathways in association with RT. The present study was designed in a similar way to work published by Raben et al (2004) who combined gefitinib with vandetanib (a vasculartargeting agent) and RT. The authors reported that the triple association induced the greatest effect on tumour growth and angiogenesis. 
The present data indicate that the double combination, the concomitant application of the VEGF signalling inhibitor, AZD2171, with gefitinib produced more than additive effects with a CR value of 1.6 , and that the triple combination, the concomitant

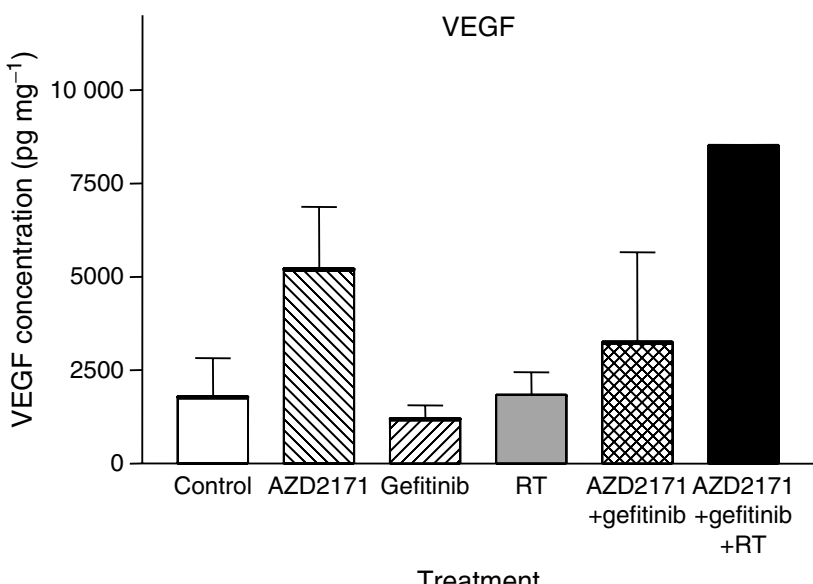

Figure 7 Human VEGF tumour concentration ( $\mathrm{pg} \mathrm{mg}^{-1}$ protein) on day 24 at the end of the treatment period for the different treatments (mean concentration \pm s.d.), three tumours for controls and AZD2I7I, four tumours for conditions with gefitinib, RT and AZD2 I7I - gefitinib; the triple combination could be analysed on one tumour only, due to the very small size of the tumours reflecting the efficiency of the combined treatment. application of the VEGF signalling inhibitor, AZD2171-gefitinib association with $\mathrm{RT}$, produced the highest supra-additive antitumour effects with a $\mathrm{CR}$ value of 2 . Importantly, this triple combination maintained a longer growth delay in comparison either to AZD2171-gefitinib double combination or to RT. The exploration of several tumour markers sustained the antitumour effects, which were observed. Among them, it was interesting to note that the antitumour efficacy of the triple combination could be attributed both to a direct impact on tumour cell proliferation (Ki67 with a marked decrease in labelling) and on the endothelial cell network of the tumour (vWF staining). A previous experimental study by our group was performed on an immortalised microvascular endothelial cell line of human origin (Bozec et al, 2005). This cell line was exposed to a combination, including gefitinib, with ZM317450 a tyrosine kinase inhibitor against VEGFR-2 and RT. A marked synergistic interaction for cytotoxic effect was found with this triple combination only. These data concur well with the present results showing a decrease of the number of endothelial cells, as indicated by vWF staining, by combining AZD2171 with gefitinib and RT compared to untreated controls and either monotherapy. It was not within the scope of the present study to examine the effects of the respective associations between single drugs and RT. The AZD2171-gefitinib combination was taken as a whole when combined with RT.

Blood plasma levels of VEGF are significantly increased by VEGFR-2 blockade in mice and were proposed as a surrogate marker for VEGFR-2 targeted therapy in the clinical situation (Jain et al, 2006). Sunitinib, a tyrosine kinase inhibitor of VEGFRs, was shown to increase plasma levels of VEGF in treated patients (Faivre
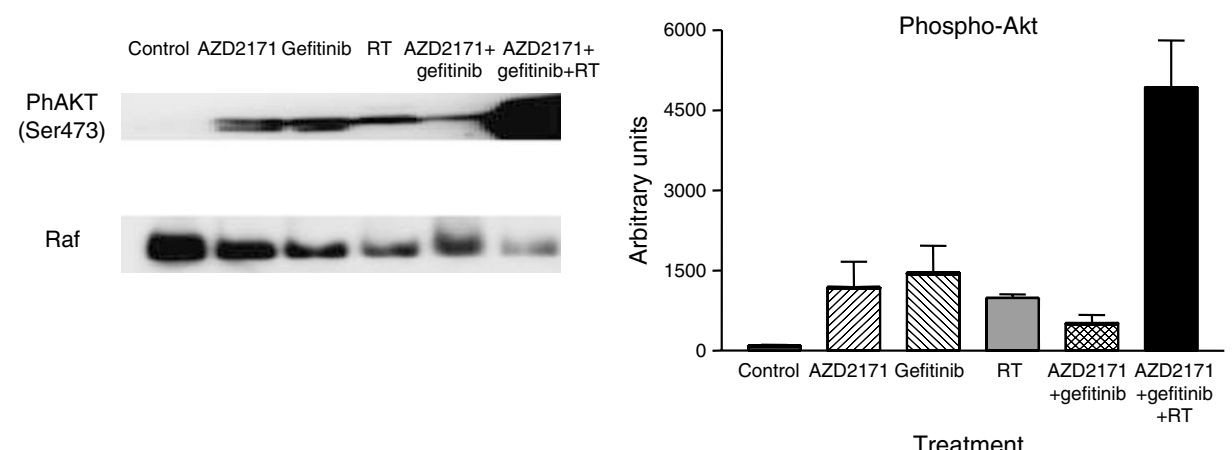

Figure 8 Phospho-AKT expression for tumours in regrowth following different treatments. Tumours collected when mean tumour volume in each group reached $2500 \mathrm{~mm}^{3}$ (mean \pm s.d., three tumours for each treatment condition). A typical example of Western blot analysis is given. The results were normalised vs Raf taken as a loading control.

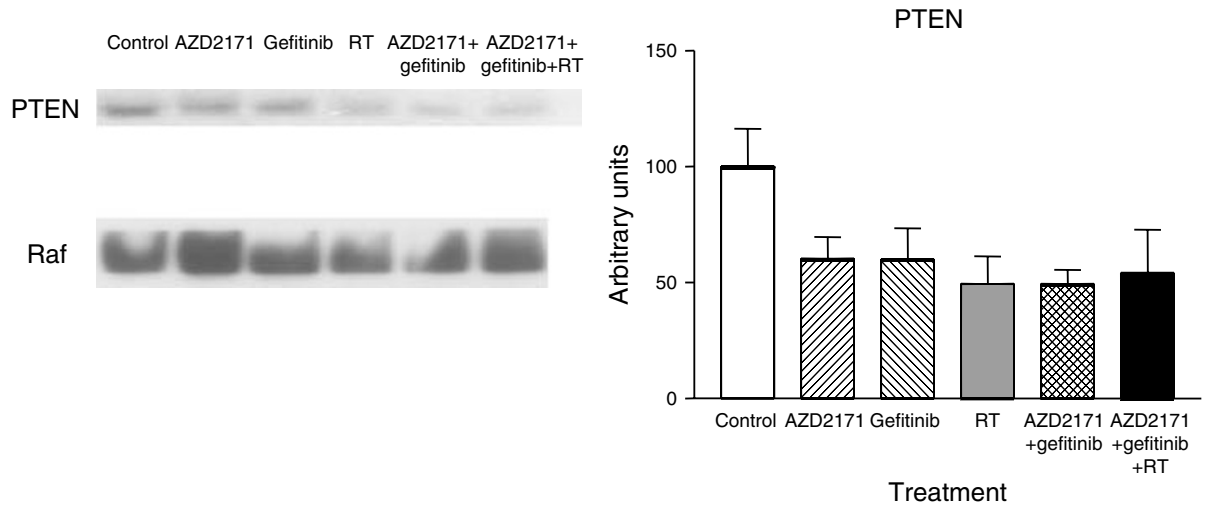

Figure 9 PTEN expression for tumours in regrowth following the different treatments. Tumours collected at the end of follow-up when mean tumour volume in each group reached $2500 \mathrm{~mm}^{3}$ (mean \pm s.d., three tumours for each treatment condition). A typical example of Western blot analysis is given. The results were normalised vs Raf taken as a loading control. 

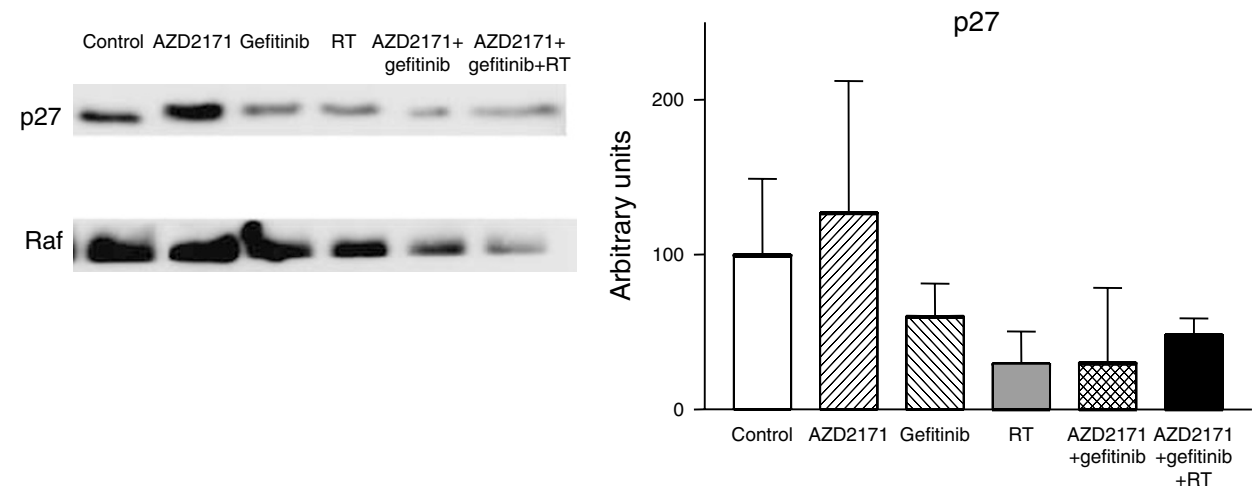

Treatment

Figure 10 p27 expression for tumours in regrowth following the different treatments. Tumours collected when mean tumour volume in each group reached $2500 \mathrm{~mm}^{3}$ (mean \pm s.d., three tumours for each treatment condition). A typical example of Western blot analysis is given. The results were normalised vs Raf taken as a loading control.

et al, 2006a). The present data indicate that AZD2171, a highly potent VEGFR-2 inhibitor, was able to increase VEGF tumour expression. The strict tumour origin of the measured VEGF was based on the fact that the measured VEGF was of human origin. This observation confirms that changes in VEGF levels under treatment may be the reflect of modifications occurring in tumour VEGF expression.

One of the molecular explanations for the supra-additive effects on tumour growth presently observed may lie in the changes in tumour expression of the DNA repair protein ERCC1. As expected, ERCC1 expression was markedly enhanced by RT treatment alone. When combined with AZD2171 and gefitinib, this RT-dependent induction of ERCC1 was totally abrogated (Figure 6).

PTEN inactivation and constitutive activation of AKT are well-defined genetic alterations in the initiation and progression of tumours (Vivanco and Sawyers, 2002). AKT is a crucial survival kinase, and interfering with AKT expression is an attractive strategy to control tumour progression (Goswami et al, 2006). Interestingly, the present data show that tumour regrowth was accompanied by marked changes in tumour expression of PTEN and phospho-AKT. PTEN was found to be significantly decreased. Conversely, phospho-AKT expression was enhanced as compared to controls. This was particularly true in tumours having shown the highest response under treatment (with the triple combination), AKT is one of the main mTOR-related messengers (Smolewski, 2006) and the current development of mTOR inhibitors as anticancer drugs is very promising (Faivre et al, 2006b). It is suggested that to add mTOR targeting as an additional sequence of treatment following the presently studied combination could maintain prolonged antitumour effects over time. Overall, the present data highlight the interesting antitumour effects of the triple combination with gefitinib, AZD2171 and RT. This strategy could serve as a basis for future innovative clinical trials.

\section{REFERENCES}

Bozec A, Formento P, Ciccolini J, Fanciullino R, Padovani L, Murraciole X, Fischel JL, Milano G (2005) Response of endothelial cells to a dual tyrosine kinase receptor inhibition combined with irradiation. Mol Cancer Ther 4: $1962-1971$

Cao C, Albert JM, Geng L, Ivy PS, Sandler A, Johnson DH, Lu B (2006) Vascular endothelial growth factor tyrosine kinase inhibitor AZD2171 and fractionated radiotherapy in mouse models of lung cancer. Cancer Res 66: $11409-11415$

Ciardiello F, Bianco R, Damiano V, Fontanini G, Caputo R, Pomatico G, De Placido S, Bianco AR, Mendelsohn J, Tortora G (2000) Anti-angiogenic and antitumour activity of antiepidermal growth factor receptor C225 monoclonal antibody in combination with vascular endothelial growth factor antisense oligonucleotides in human GEO colon cancer cells. Clin Cancer Res 6: 3739-3747

Ciardiello F, Caputo R, Bianco R, Damiano V, Fontanini G, Cuccato S, De Placido S, Bianco AR, Tortora G (2001) Inhibition of growth factor production and angiogenesis in human cancer cells by ZD1839 (Iressa), a selective epidermal growth factor receptor tyrosine kinase inhibitor. Clin Cancer Res 7: 1459-1465

Dassonville O, Formento JL, Francoual M, Ramaioli A, Santini J, Schneider M, Demard F, Milano G (1993) Expression of epidermal growth factor receptor and survival in upper aerodigestive tract cancer. J Clin Oncol 11: $1873-1878$

Faivre S, Delbado C, Vera K, Robert C, Lozahic S, Lassau N, Bello C, Deprimo S, Brega N, Massimini G, Armand JP, Scigalla P, Raymond E (2006a) Safety, pharmacokinetic and antitumor activity of SI11248, a novel oral multitarget tyrosine kinase inhibitor, in patients with cancer. J Clin Oncol 24: 25-35
Faivre S, Kroemer G, Raymond E (2006b) Current development of mTOR inhibitors as anticancer agents. Nat Rev Drug Discov 5: 671-688

Goswami A, Ranganathan P, Rangnekar V (2006) The phosphoinositide 3-kinase/Akt1/Par-4 axis: a cancer-selective therapeutic target. Cancer Res 66: $2889-2892$

Herbst RS, Johnson DH, Mininberg E, Carbone DP, Henderson T, Kim ES, Blumenschein Jr G, Lee JJ, Liu DD, Truong MT, Hong WK, Tran H, Tsao A, Xie D, Ramies DA, Mass R, Seshagiri S, Eberhard DA, Kelley SK, Sandler A (2005) Phase I/II trial evaluating the anti-vascular endothelial growth factor monoclonal antibody bevacizumab in combination with the HER-1/epidermal growth factor receptor tyrosine kinase inhibitor erlotinib for patients with recurrent non-small-cell lung cancer. J Clin Oncol 23: $2544-2555$

Herbst RS, Mininberg E, Henderson T, Kim E, Hong WK, Mass R, Novotny W, Garcia B, Johnson D, Sandler A (2003) Phase I/II trial evaluating blockade of tumour blood supply and tumour cell proliferation with combined bevacizumab and erlotinib HCI as targeted cancer therapy in patients with recurrent non-small cell lung cancer. Eur J Cancer 1: S293 Hirata A, Ogawa S, Kometani T, Kuwano T, Naito S, Kuwano M, Ono M (2002) ZD1839 (Iressa) induces antiangiogenic effects through inhibition of epidermal growth factor receptor tyrosine kinase. Cancer Res 62: $2554-2560$

Jain RK, Duda DG, Clark JW, Loeffler JS (2006) Lessons from phase III clinical trials on anti-VEGF therapy for cancer. Nat Clin Pract Oncol 3: $24-40$

Jung YD, Mansfield PF, Akagi M, Takeda A, Liu W, Bucana CD, Hicklin DJ, Ellis LM (2002) Effects of combination anti-vascular endothelial growth 
factor receptor and anti-epidermal growth factor receptor therapies on the growth of gastric cancer in a nude mouse model. Eur J Cancer 38: $1133-1140$

Le QT, Giaccia AJ (2003) Therapeutic exploitation of the physiological and molecular genetic alterations in head-and-neck cancer. Clin Cancer Res 9 $4287-4295$

Nieder C, Wiedenmann N, Andratschke N, Molls M (2006) Current status of angiogenesis inhibitors combined with radiation therapy. Cancer Treat Rev 32: $348-364$

Perrotte P, Matsumoto T, Inoue K, Kuniyasu H, Eve BY, Hicklin DJ, Radinsky R, Dinney CP (1999) Anti-epidermal growth factor receptor antibody C225 inhibits angiogenesis in human transitional cell carcinoma growing orthotopically in nude mice. Clin Cancer Res 2: $257-265$

Prewett MC, Hooper AT, Bassi R (2002) Enhanced antitumour activity of anti-epidermal growth factor receptor monoclonal antibody IMC-C225 in combination with irinotecan (CPT-11) against human colorectal tumour xenografts. Clin Cancer Res 8: 994-1003

Raben D, Bianco C, Damiano V, Bianco R, Melisi D, Mignogna C, D'Armiento FP, Cionini L, Bianco AR, Tortora G, Ciardiello F, Bunn P (2004) Antitumour activity of ZD6126, a novel vascular-targeting agent, is enhanced when combined with ZD183, an epidermal growth factor receptor tyrosine kinase inhibitor, and potentiates the effects of radiation in a human non-small cell lung cancer xenograft model. Mol Cancer Ther 3: $977-983$

Simon R, Mirlacher M, Sauter G (2004) Tissue microarrays. Biotechniques 36: $98-105$
Smolewski P (2006) Recent developments in targeting the mammalian target of rapamycin (mTOR) kinase pathway. Anticancer Drugs 17: $487-494$

Vivanco I, Sawyers CL (2002) The phosphatidylinositol 3-kinase AKT pathway in human cancer. Nat Rev Cancer 2: 489-501

Wachsberger PR, Burd R, Marero N, Daskalakis C, Ryan A, McCue P, Dicker AP (2005) Effect of the tumour vascular-damaging agent, ZD6126, on the radioresponse of U87 glioblastoma. Clin Cancer Res 11: $835-842$

Wedge SR, Kendrew J, Hennequin LF, Valentine PJ, Barry ST, Brave SR, Smith NR, James NH, Dukes M, Curwen JO, Chester R, Jackson JA, Boffey SJ, Kilburn LL, Barnett S, Richmond GH, Wadsworth PF, Walker M, Bigley AL, Taylor ST, Cooper L, Beck S, Jürgensmeier JM, Ogilvie DJ (2005) AZD2171: a highly potent, orally bioavailable, vascular endothelial growth factor receptor-2 tyrosine kinase inhibitor for the treatment of cancer. Cancer Res 65: 4389-4400

Williams KJ, Telfer BA, Shannon AM, Babur M, Stratford IJ, Wedge SR (2007) Combining radiotherapy with AZD2171, a potent inhibitor, of vascular endothelial growth factor signaling: pathophysiologic effects and therapeutic benefit. Mol Cancer Ther 6: 599-606

Woodburn JR (1999) The epidermal growth factor receptor and its inhibition in cancer therapy. Pharmacol Ther 82: 241-250

Workman P, Twentyman P, Balkwill F, Balmain A, Chaplin D, Double J, Embleton J, Newell D, Raymond R, Stables J, Stephens T, Wallace J (1998) United Kingdom Co-ordinating Committee on Cancer Research (UKCCCR) guidelines for the welfare of animals in experimental neoplasia (second edition). Br J Cancer 77: 1-10 